UTHEP-521

hep-th/0604103

April 2006

\title{
Bosonic Massless Higher Spin Fields from Matrix Model
}

\author{
Takashi Saitou ${ }^{1}$ \\ Institute of Physics, University of Tsukuba, \\ Tsukuba, Ibaraki 305-8571, Japan
}

\begin{abstract}
We study matrix models as a new approach to formulate massless higher spin gauge field theory. As a first step in this direction, we show that the free equation of motion of bosonic massless higher spin gauge fields can be derived from that of a matrix model.
\end{abstract}

\footnotetext{
${ }^{1}$ e-mail: saitout@het.ph.tsukuba.ac.jp
} 


\section{Introduction}

It has been known that there are problems in the construction of consistent interactions for massless higher spin gauge fields, though there are physically acceptable free field Lagrangian for them. Free field Lagrangian for higher spin fields, in which these particles are expressed by totally symmetric tensor or tensor-spinor fields, was originally derived by Fronsdal for bosons [2] and Fang-Fronsdal for fermions [3]. ${ }^{2}$ There are some approaches for the construction of free field Lagrangian 4] [5. Interaction problems appear when one tries to couple massless higher spin fields to an electromagnetic field [6, to gravity [7] 8] 9] or to construct selfinteractions [10] 11]. For a review of massless higher spin gauge field theory, see [12].

At present, there exist various approaches to the theory, which solve the interaction problems in some cases. For example, an approach, called the unfolded formalism, was developed by Vasiliev et al [13] [14. They succeeded in the construction of interacting higher spin gauge theory with a nonzero cosmological constant 15. An approach, called BRST approach, was initiated by development of string field theory on the basis of BRST techniques [16] [17] [18, [19] 20].

In this paper, we study matrix models as a new approach to formulate massless higher spin gauge field theory. Recently, it has been shown that the Einstein equation can be obtained from the equation of motion of a matrix model by introducing a new interpretation of the matrix model, in which matrices represent differential operators on a curved spacetime 1]. Furthermore, it was pointed out that there is a possibility that matrix models include the degrees of freedom of massless higher spin gauge fields. An advantage of this formalism is that the matrix model possesses a gauge invariance manifestly, embedded in the $U(N)$ symmetry. Therefore it is interesting to analyze interacting massless higher spin gauge field theory using the matrix model.

A first step towards constructing massless higher spin gauge field theory is the formulation of the free theory. Therefore, in this paper we show that the free equation of motion of bosonic massless higher spin gauge fields can be derived from that of the matrix model.

There is another motivation for our study. Massless higher spin fields are expected to appear in the tensionless limit of string theory, since mass squared of them are all proportional to the string tension. Thus in this limit, one should observe an enhancement of gauge sym-

\footnotetext{
${ }^{2}$ In four dimensional spacetime, all higher spin fields can be described either by totally symmetric tensor or totally symmetric tensor-spinor fields. This is not the case for a dimension that is larger than four. In this paper, we will restrict ourselves to the consideration of symmetric tensor fields.
} 
metry of string theory by that of the massless higher spin field theory. On the other hand, matrix models are expected to be a nonperturbative formulation of string theory. Therefore our study may be useful for better understanding of gauge symmetry of string theory and can lead to further understanding of nonperturbative aspects of string theory.

The organization of this paper is as follows. In section 2, we briefly review the results of [1. In section 3, we show that the free equation of motion of bosonic massless higher spin gauge fields can be derived from that of the matrix model. Section 4 is devoted to conclusions and future works.

\section{Matrix Model}

In this section, we briefly review the results of [1]. Introducing a new interpretation of a matrix model, we will see the following three facts.

- Vacuum Einstein equation can be derived from the equation of motion of the matrix model.

- There is a possibility that the matrix model can describe bosonic massless higher spin fields.

- Gauge symmetries related to higher spin fields are embedded in the $U(N)$ symmetry of the matrix model.

In this paper, we consider the large $N$ reduced model of $D$-dimensional pure Yang-Mills theory with $U(N)$ gauge symmetry as the matrix model :

$$
S=-\frac{1}{4 g^{2}} \operatorname{tr}\left(\left[A_{a}, A_{b}\right]\left[A^{a}, A^{b}\right]\right)
$$

where $A_{a}$ are $N \times N$ hermitian bosonic matrices. Latin indices denote Euclidean spacetime directions. This action has the $S O(D)$ Lorentz symmetry and $N \times N$ unitary matrix symmetry. We can also consider supersymmetric version of this model, but in this paper we consider only the bosonic action (11). ${ }^{3}$

The basic idea of [1] is that matrices represent differential operators on a curved spacetime. There are several problems with this identification. For example, matrices act as

\footnotetext{
${ }^{3}$ In $D=10$, supersymmetric version of the action (11) is nothing but the action of IIB matrix model [21].
} 
Endomorphisms on a vector space, which means matrices map a vector space to itself. On the contrary, covariant derivatives map a tensor field of rank- $n$ to a tensor field of rank$(n+1)$. Therefore we should prepare a vector space $V$ which contain at least tensor fields of any rank and prepare an object $\nabla_{(a)}$ which is equivalent to a covariant derivative $\nabla_{a}$ such that each component of $\nabla_{(a)}$ is expressed as an Endomorphism on $V$, in order to interpret covariant derivatives as matrices. In [1] the authors showed that $V$ can be given by the space of smooth functions on the principal $\operatorname{Spin}(D)$ bundle over a manifold $M$. A smooth function $f$ on it is defined as the mapping

$$
f: U \times \operatorname{Spin}(D) \rightarrow \mathbb{C}
$$

where $U$ denotes a patch on $M$. Thus, $f$ depends on the local coordinate $(x, g)$, where $x \in U$ and $g \in \operatorname{Spin}(D)$, of the principal $\operatorname{Spin}(D)$ bundle over $M . \nabla_{(a)}$ can be given by

$$
\nabla_{(a)}=R_{(a)}^{b}\left(g^{-1}\right) \nabla_{b}
$$

where $R_{(a)}{ }^{b}(g)$ is the vector representation of $\operatorname{Spin}(D) .{ }^{4}$ The covariant derivative $\nabla_{a}$ is defined as

$$
\nabla_{a}=e_{a}^{\mu}(x)\left(\partial_{\mu}+\omega_{\mu}^{b c}(x) \varnothing_{b c}\right)
$$

where $\varnothing_{b c}\left(=-\varnothing_{c b}\right)$ is the generator of the local Lorentz group $\operatorname{Spin}(D), e_{a}{ }^{\mu}(x)$ is the vierbein and $\omega_{\mu}{ }^{a b}(x)$ is the spin-connection. Here, Latin indices denote the local Lorentz indices. Notice that $\nabla_{a}$ maps a rank- $n$ tensor to a rank- $(n+1)$ tensor and $\varnothing_{a b}$ acts on the local Lorentz indices of these tensors. Therefore we have

$$
\left[\varnothing_{a b}, \nabla_{c}\right]=\frac{1}{2}\left(\delta_{a c} \nabla_{b}-\delta_{b c} \nabla_{a}\right)
$$

in this setting, which will be used later.

Let us see how the Einstein equation can be derived from the equation of motion of the matrix model by applying this interpretation to the matrix model. From the action (11), we obtain the following equation of motion :

$$
\left[A^{a},\left[A_{a}, A_{b}\right]\right]=0
$$

\footnotetext{
${ }^{4}$ Though $R_{a}{ }^{b}(g)$ and $R_{(a)}{ }^{b}(g)$ are the same quantity, we distinguish them because indices $a$ is transformed by the action of $G$, while $(a)$ is not.
} 
We substitute $A_{a}=i \nabla_{a}$ into the equation of motion (6), where $i$ is introduced to make $A_{a}$ hermitian. ${ }^{5}$ The commutator of $A_{a}$ becomes

$$
\begin{aligned}
{\left[A_{a}, A_{b}\right] } & =\left[i e_{a}{ }^{\mu} \nabla_{\mu}, i e_{b}{ }^{\nu} \nabla_{\nu}\right] \\
& =-e_{a}{ }^{\mu} e_{b}{ }^{\nu}\left[\nabla_{\mu}, \nabla_{\nu}\right]-e_{[a}{ }^{\mu}\left(\nabla_{\mu} e_{b]}{ }^{\nu}\right) \nabla_{\nu} \\
& =-R_{a b}{ }^{c d} \emptyset_{c d}-C^{\nu}{ }_{a b} \nabla_{\nu}
\end{aligned}
$$

where $R_{a b, c d}$ is the Riemann tensor and $C^{\nu}{ }_{a b}$ is the torsion tensor. Here, we put the torsionless constraint $C^{\nu}{ }_{a b}=0$ to solve the spin-connection in terms of the vierbein. Under the torsionless constraint, the equation of motion becomes

$$
\begin{aligned}
{\left[A^{a},\left[A_{a}, A_{b}\right]\right] } & =\left[i \nabla^{a},-R_{a b}{ }^{c d} \mathcal{O}_{c d}\right] \\
& =-i\left[\nabla^{a}, R_{a b}{ }^{c d}\right] \mathcal{O}_{c d}-i R_{a b}{ }^{c d}\left[\nabla^{a}, \mathcal{O}_{c d}\right] \\
& =-i\left(\nabla^{a} R_{a b}{ }^{c d}\right) \mathcal{O}_{c d}+i R_{b}{ }^{c} \nabla_{c}=0
\end{aligned}
$$

and we obtain the following two equations :

$$
R_{b c}=0, \quad \nabla^{a} R_{a b, c d}=0
$$

The first equation is the vacuum Einstein equation. The second equation can be derived from the first one and the Bianchi identity : $\nabla_{a} R_{d e}^{b c}+\nabla_{b} R_{d e}^{c a}+\nabla_{c} R_{d e}^{a b}=0$. Thus, we have obtained the Einstein equation from the equation of motion of the matrix model.

Since each component of matrices $A_{a}$ acts functions on the principal $S O(D)$ bundle over $M$ as an Endomorphism, in general, $A_{a}$ may be expanded as

$$
A_{a}=i \nabla_{a}+a_{a}(x)+\frac{i}{2}\left\{b_{a}{ }^{b}(x), \nabla_{b}\right\}+\frac{i}{2}\left\{B_{a}{ }^{b c}(x), \varnothing_{b c}\right\}+\frac{i^{2}}{2}\left\{e_{a}^{b c}(x), \nabla_{b} \nabla_{c}\right\}+\cdots,
$$

where $i$ and anticommutator \{\} are introduced to make $A_{a}$ hermitian. The coefficient $e_{a}{ }^{b c}(x)$ can be taken to be symmetric under exchange of the indices $b \leftrightarrow c$ because antisymmetric part can be absorbed in the term that is the first order in $\varnothing_{a b}$. Higher order terms expanded in terms of the operators $\nabla_{a}$ and $\varnothing_{a b}$ also can be taken to be symmetric under permutations of the operators. We consider the expansion as a sum of homogeneous polynomials in $\nabla_{a}$ and $\varnothing_{a b}$, whose coefficients are identified with massless higher spin gauge fields. Coefficients of the first order homogeneous polynomial express spin-2 gauge fields, and those of the

\footnotetext{
${ }^{5}$ Hermiticity can be confirmed by defining the inner product $(u, v)=\int e d^{d} x d g u^{*}(x, g) v(x, g)$, where $e=\operatorname{det}\left(e_{a}{ }^{\mu}\right), d g$ is a Haar measure of group $G, u(x, g)$ and $v(x, g)$ are arbitrary functions of $x$ and $g \in G$
} 
second order one express spin-3 gauge fields and so on. Thus, the number of independent components of higher spin gauge fields grows rapidly with degree in $\nabla_{a}$ and $\varnothing_{a b}$.

Here, we mention how gauge symmetries are embedded in the $U(N)$ symmetry of the matrix model. Originally, the $U(N)$ symmetry of the matrix model is written as

$$
\delta A_{a}=i\left[\Lambda, A_{a}\right]
$$

where $\Lambda$ is a $N \times N$ hermitian matrix. In the new interpretation of the matrix model, $\Lambda$ becomes a scalar operator expanded in terms of $\nabla_{a}$ and $\varnothing_{a b}$.

Let us see how gauge transformations are generated by $\Lambda$ in the case of spin-3. In order to deal with this case, we need to keep track of only the first and the fifth term in (10),

$$
A_{a}=i \nabla_{a}+(i)^{2}\left\{e_{a}{ }^{a_{1} a_{2}}(x), \nabla_{a_{1}} \nabla_{a_{2}}\right\}+\cdots .
$$

We take $\Lambda$ as

$$
\Lambda=(i)^{2}\left\{\lambda^{a_{1} a_{2}}(x), \nabla_{a_{1}} \nabla_{a_{2}}\right\}
$$

Then (11) becomes

$$
\begin{aligned}
\delta A_{a} & =i\left[\Lambda, A_{a}\right] \\
& =\left(\nabla_{a} \lambda^{a_{1} a_{2}}(x)\right) \nabla_{a_{1}} \nabla_{a_{2}}+\cdots .
\end{aligned}
$$

Thus $e_{a}{ }^{a_{1} a_{2}}(x)$ transforms as

$$
\delta e_{a}{ }^{a_{1} a_{2}}(x)=\nabla_{a} \lambda^{a_{1} a_{2}}(x)+\cdots .
$$

Therefore, $e_{a}{ }^{a_{1} a_{2}}(x)$ transforms as a rank-3 symmetric tensor field. Other gauge transformations related to higher spin gauge fields are realized in terms of other $\Lambda$. Thus, this formulation possesses the gauge invariance related to higher spin gauge fields manifestly. This is an advantage of this formulation.

\section{Free Higher Spin Field Equation in Flat Spacetime}

The free equation of motion for the rank-s totally symmetric tensor field $\phi_{\mu_{1} \cdots \mu_{s}}$ in $D$ dimensional flat spacetime, the so-called "Fronsdal equation", is given by

$$
W_{\mu_{1} \cdots \mu_{s}} \equiv \square \phi_{\mu_{1} \cdots \mu_{s}}-s \partial_{\left(\mu_{1}\right.}(\partial \cdot \phi)_{\left.\mu_{2} \cdots \mu_{s}\right)}+s(s-1) \partial_{\left(\mu_{1}\right.} \partial_{\mu_{2}} \phi_{\left.\mu_{3} \cdots \mu_{s}\right)}^{\prime}=0,
$$


where we use notations $(\partial \cdot \phi)_{\mu_{1} \cdots \mu_{s-1}}=\partial_{\rho} \phi^{\rho}{ }_{\mu_{1} \cdots \mu_{s-1}}$ and $\phi_{\mu_{1} \cdots \mu_{s-2}}^{\prime}=\phi_{\rho \mu_{1} \cdots \mu_{s-2}}^{\rho}$. Greek indices run from 1 to $D$ and denote flat spacetime directions. This equation of motion possesses the gauge symmetry, the so-called Fronsdal symmetry,

$$
\delta \phi_{\mu_{1} \cdots \mu_{s}}=\partial_{\left(\mu_{1}\right.} \lambda_{\left.\mu_{2} \cdots \mu_{s}\right)},
$$

where the bracket () denotes symmetrization of the flat spacetime indices and the gauge parameter $\lambda_{\mu_{1} \cdots \mu_{s-1}}$ is symmetric under permutations of the indices. The conventional formulation for free totally symmetric tensor gauge fields was originally derived by Fronsdal 2]. The key feature of this formulation is the need for a pair of constraints, one on the parameter $\lambda_{\mu_{1} \cdots \mu_{s-1}}$, whose trace $\lambda^{\rho}{ }_{\rho \mu_{1} \cdots \mu_{s-3}}=\lambda_{\mu_{1} \cdots \mu_{s-3}}^{\prime}$ is required to vanish, and one on the gauge field itself, whose double trace $\phi_{\rho}^{\rho}{ }_{\rho}^{\sigma} \sigma \mu_{1} \cdots \mu_{s-5}=\phi_{\mu_{1} \cdots \mu_{s-5}}^{\prime \prime}$ is also required to vanish.

In this section, we show that the free equation of motion of higher spin gauge fields (16) can be derived from that of the matrix model (6). In our formulation, as we will see in the next subsection, the constraints on $\phi_{\mu_{1} \cdots \mu_{s}}$ are achieved by putting traceless constraints on the fields in $A_{a}$. Through the analysis in this section, we can understand how higher spin gauge fields are included in the matrix model.

We show the case of spin-3 in subsection 3.1 and spin- $s$ in subsection 3.2.

\subsection{Free Spin-3 Field Equation in Flat Spacetime}

Let us first consider the spin-3 case as an example. The equation of motion of spin-3 field $\phi_{\mu \nu \rho}$ is given by

$$
\begin{array}{r}
W_{\mu \nu \rho} \equiv \square \phi_{\mu \nu \rho}-\partial_{\mu}(\partial \cdot \phi)_{\nu \rho}-\partial_{\nu}(\partial \cdot \phi)_{\rho \mu}-\partial_{\rho}(\partial \cdot \phi)_{\mu \nu} \\
+\partial_{\mu} \partial_{\nu} \phi_{\rho}^{\prime}+\partial_{\nu} \partial_{\rho} \phi_{\mu}^{\prime}+\partial_{\rho} \partial_{\mu} \phi_{\nu}^{\prime}=0 .
\end{array}
$$

In this and the next subsection, in order to derive free field equations we keep only terms linear with respect to the component fields and we use the ordinary differential operator $\partial_{\mu}$ instead of the covariant derivative $\nabla_{a}$. Notice that the commutation relation of the operators is given by

$$
\left[\emptyset_{\mu \nu}, \partial_{\rho}\right]=\frac{1}{2}\left(\delta_{\mu \rho} \partial_{\nu}-\delta_{\nu \rho} \partial_{\mu}\right) .
$$


In order to deal with the spin-3 case, we keep track of the second order homogeneous polynomial of the operators $\partial_{\mu}$ and $\varnothing_{\mu \nu}$ in $A_{\mu}$ :

$$
\begin{array}{r}
A_{\mu}=i \partial_{\mu}+i^{2} e_{\mu,}{ }^{\nu \rho}(x) \\
\partial_{\nu} \partial_{\rho}+i^{2} \frac{1}{2} \omega_{\mu,}{ }^{\nu, \rho \sigma}(x)\left(\partial_{\nu} \varnothing_{\rho \sigma}+\varnothing_{\rho \sigma} \partial_{\nu}\right) \\
+i^{2} \frac{1}{2} \Omega_{\mu}{ }^{\rho \sigma, \lambda \kappa}(x)\left(\varnothing_{\rho \sigma} \varnothing_{\lambda \kappa}+\varnothing_{\lambda \kappa} \varnothing_{\rho \sigma}\right) .
\end{array}
$$

Here, we do not write the anticommutator appeared in (10) explicitly because terms like $\left(\partial_{\nu} e_{\mu}^{\nu \rho}\right) \partial_{\rho}$ are not needed to derive spin-3 field equation.

Based on the analogy of the frame formulation of gravity, we can regard that the fields as $e_{\mu, \nu \rho}$ and $\omega_{\mu, \nu, \rho \sigma}$ are generalizations of the vierbein and the spin-connection, respectively. We assume that the fields $e_{\mu, \nu \rho}$ and $\omega_{\mu, \nu, \rho \sigma}$ satisfy the traceless conditions

$$
e_{\mu, \rho}^{\rho}=0, \quad \omega_{\mu, \rho, \rho}^{\rho}=0 .
$$

The totally symmetric tensor field $\phi_{\mu \nu \rho}$ is defined in terms of $e_{\mu, \nu \rho}$ as

$$
\phi_{\mu \nu \rho} \equiv e_{(\mu, \nu \rho)}=\frac{1}{3}\left(e_{\mu, \nu \rho}+e_{\nu, \rho \mu}+e_{\rho, \mu \nu}\right),
$$

and $\omega_{\mu, \nu, \rho \sigma}$ and $\Omega_{\mu, \rho \sigma, \lambda \kappa}$ are auxiliary fields. As we will see shortly, the relation (22) can be understood from the gauge transformations which are embedded in the $U(N)$ symmetry of the matrix model.

Let us summarize the gauge transformations. There are three kinds of gauge transformations in the case of spin-3 :

(i) Generalized coordinate transformation, generated by $\Lambda=\lambda^{\mu \nu} \partial_{\mu} \partial_{\nu}$,

$$
\delta e_{\mu, \nu \rho}=\partial_{\mu} \lambda_{\nu \rho}, \quad \delta \omega_{\mu, \nu, \rho \sigma}=0, \quad \delta \Omega_{\mu, \rho \sigma, \lambda \kappa}=0
$$

where the parameter $\lambda_{\mu \nu}$ satisfies $\lambda_{\mu \nu}=\lambda_{\nu \mu}$.

(ii) Generalized local Lorentz transformation, generated by $\Lambda=\frac{1}{2} \lambda^{\nu, \rho \sigma}\left(\partial_{\nu} \varnothing_{\rho \sigma}+\varnothing_{\rho \sigma} \partial_{\nu}\right)$,

$$
\delta e_{\mu, \nu \rho}=\lambda_{\nu, \mu \rho}+\lambda_{\rho, \mu \nu} \equiv \Lambda_{\mu, \nu \rho}, \quad \delta \omega_{\mu, \nu, \rho \sigma}=\partial_{\mu} \lambda_{\nu, \rho \sigma}, \quad \delta \Omega_{\mu, \rho \sigma, \lambda \kappa}=0
$$

where the parameter $\lambda_{\mu, \nu \rho}$ satisfies $\lambda_{\mu, \nu \rho}=-\lambda_{\mu, \rho \nu}$.

(iii) Auxiliary gauge transformation, generated by $\Lambda=\frac{1}{2} \lambda^{\rho \sigma, \lambda \kappa}\left(\varnothing_{\rho \sigma} \varnothing_{\lambda \kappa}+\varnothing_{\lambda \kappa} \varnothing_{\rho \sigma}\right)$,

$$
\delta e_{\mu, \nu \rho}=0, \quad \delta \omega_{\mu, \nu, \rho \sigma}=\lambda_{\mu \nu, \rho \sigma}+\lambda_{\rho \sigma, \mu \nu}, \quad \delta \Omega_{\mu, \rho \sigma, \lambda \kappa}=\partial_{\mu} \lambda_{\rho \sigma, \lambda \kappa} .
$$

where the parameter $\lambda_{\mu \nu, \rho \sigma}$ satisfies $\lambda_{\mu \nu, \rho \sigma}=-\lambda_{\nu \mu, \rho \sigma}=-\lambda_{\mu \nu, \sigma \rho}$. 
Based on the analogy of the frame formulation of gravity, the gauge transformation (i), which corresponds to the Fronsdal symmetry, is an extension of generalized coordinate transformation. The gauge transformation (ii), which removes the part that is not totally symmetric in the indices of $e_{\mu, \nu \rho}$, is an extension of local Lorentz transformation. The gauge symmetry (iii), which does not act on dynamical fields, appears only for spins larger than 2.

Under the gauge transformation (i), the rank-3 totally symmetric tensor field $\phi_{\mu \nu \rho}$ defined in (22) transforms as follows :

$$
\delta \phi_{\mu \nu \rho}=\partial_{\mu} \lambda_{\nu \rho}+\partial_{\nu} \lambda_{\rho \mu}+\partial_{\rho} \lambda_{\mu \nu}
$$

This is consistent with the identification (22).

Since the gauge transformation (i) corresponds to the Fronsdal one, in order to derive higher spin field equations we should fix the gauge symmetries (ii) and (iii). However, gauge symmetries cannot remove all extra degrees of freedom. Therefore, we must impose some constraints that can be used to determine the auxiliary fields $\omega_{\mu, \nu, \rho \sigma}$ and $\Omega_{\mu, \rho \sigma, \lambda \kappa}$ in terms of the dynamical field $e_{\mu, \nu \rho}$. In the rest of this subsection, we will perform these procedures in order.

\section{Constraints}

Based on the analogy of the frame formulation of gravity, we impose constraints on the field strengths in order to solve the auxiliary the fields $\omega_{\mu, \nu, \rho \sigma}$ and $\Omega_{\mu, \rho \sigma, \lambda \kappa}$ in terms of $e_{\mu, \nu \rho}$. The field strengths are coefficients of the operators in the commutator of $A_{\mu}$. The commutator is calculated as follows :

$$
\begin{aligned}
& {\left[A_{\mu}, A_{\nu}\right]=-i \partial_{[\mu} e_{\nu]}{ }^{\rho \sigma} \partial_{\rho} \partial_{\sigma}-\frac{i}{2} \partial_{[\mu} \omega_{\nu]}{ }^{\rho, \sigma \lambda}\left(\partial_{\rho} \varnothing_{\sigma \lambda}+\varnothing_{\sigma \lambda} \partial_{\rho}\right)} \\
& -\frac{i}{2} \Omega_{[\nu,}^{\rho \sigma, \lambda \kappa}\left[\partial_{[\mu},\left(\varnothing_{\rho \sigma} \varnothing_{\lambda \kappa}+O_{\lambda \kappa} \varnothing_{\rho \sigma}\right)\right] \\
& -\frac{i}{2} \partial_{[\mu} \Omega_{\nu]},{ }^{\rho \sigma, \lambda \kappa}\left(\varnothing_{\rho \sigma} \varnothing_{\lambda \kappa}+O_{\lambda \kappa} \varnothing_{\rho \sigma}\right) \\
& -\frac{i}{2} \Omega_{[\nu,}{ }^{\rho, \sigma \lambda}\left[\partial_{\mu]}, \partial_{\rho} \varnothing_{\sigma \lambda}+\varnothing_{\sigma \lambda} \partial_{\rho}\right] \\
& =-i\left(\partial_{[\mu} e_{\nu]},{ }^{\rho \sigma}+\omega_{[\nu,}^{\rho, \sigma}{ }_{\mu]}\right) \partial_{\rho} \partial_{\sigma} \\
& -\frac{i}{2}\left(\partial_{[\mu} \omega_{\nu],}^{\rho, \sigma \lambda}+\Omega_{[\mu, \nu]}^{\rho, \sigma \lambda}+\Omega_{[\mu,}^{\sigma \lambda, \nu]}{ }^{\rho}\right)\left(\partial_{\rho} \varnothing_{\sigma \lambda}+\varnothing_{\sigma \lambda} \partial_{\rho}\right) \\
& -\frac{i}{2} \partial_{[\mu} \Omega_{\nu]}^{\rho \sigma, \lambda \kappa}\left(\varnothing_{\rho \sigma} \varnothing_{\lambda \kappa}+\varnothing_{\lambda \kappa} \varnothing_{\rho \sigma}\right) \text {, }
\end{aligned}
$$

where the bracket [] denotes antisymmetrization of indices. We impose constraints that the 
coefficients of $\partial^{2}$ and $\varnothing^{2}$ are equal to 0 :

$$
\begin{aligned}
\partial_{[\mu} e_{\nu]}{ }^{\rho \sigma}+\omega_{[\nu,}{ }^{\rho, \sigma}{ }_{\mu]} & =0, \\
\partial_{[\mu} \Omega_{\nu], \rho \sigma, \lambda \kappa} & =0 .
\end{aligned}
$$

From (28), $\omega_{\mu, \nu, \rho \sigma}$ is solved in terms of the first order derivatives in $e_{\mu, \nu \rho}$ :

$$
\begin{aligned}
\omega_{\mu, \nu, \rho \sigma}= & \frac{1}{2} \partial_{\rho}\left(e_{\mu, \nu \sigma}+e_{\sigma, \nu \mu}\right) \\
& -\frac{1}{2} \partial_{\sigma}\left(e_{\rho, \nu \mu}+e_{\mu, \nu \rho}\right) \\
& +\frac{1}{2} \partial_{\mu}\left(e_{\sigma, \rho \nu}-e_{\rho, \nu \sigma}\right) .
\end{aligned}
$$

This is similar to the torsionless constraint of gravity. The constraint on $\Omega_{\mu, \rho \sigma, \lambda \kappa}$ (29) implies that $\Omega_{\mu, \rho \sigma, \lambda \kappa}$ can be written as a "pure gauge" configuration, ${ }^{6}$

$$
\Omega_{\mu, \rho \sigma, \lambda \kappa}=\partial_{\mu} \chi_{\rho \sigma, \lambda \kappa}
$$

where the parameter $\chi_{\rho \sigma, \lambda \kappa}$ satisfies $\chi_{\rho \sigma, \lambda \kappa}=-\chi_{\sigma \rho, \lambda \kappa}=-\chi_{\rho \sigma, \kappa \lambda}$.

Imposing these constraints, we obtain

$$
\begin{gathered}
{\left[A^{\mu},\left[A_{\mu}, A_{\nu}\right]\right]=-i\left(\partial_{[\mu} \omega_{\nu]}{ }^{\rho, \sigma \mu}+\partial_{[\mu} \chi_{\nu]}^{\rho, \sigma \mu}+\partial_{[\mu} \chi_{\nu]}^{\mu \sigma, \rho}\right) \partial_{\rho} \partial_{\sigma}} \\
-\frac{i}{2}\left[\partial^{\mu}\left(\partial_{[\mu} \omega_{\nu],}{ }^{\rho, \sigma \lambda}+\partial_{[\mu} \chi_{\nu]}{ }^{\rho, \sigma \lambda}+\partial_{[\mu} \chi^{\sigma \lambda,}{ }_{\nu]}^{\rho}\right)\right] \\
\times\left(\partial_{\rho} \varnothing_{\sigma \lambda}+\emptyset_{\sigma \lambda} \partial_{\rho}\right) .
\end{gathered}
$$

Therefore, we can obtain the following equations of motion :

$$
\begin{gathered}
\partial_{[\mu} \hat{\omega}_{\nu]},{ }^{\mu, \rho \sigma}+\partial_{[\mu} \chi_{\nu]}{ }^{(\rho, \sigma) \mu}+\partial_{[\mu} \chi_{\nu]}^{\mu(\sigma, \rho)}=0, \\
\partial^{\mu}\left(\partial_{[\mu} \omega_{\nu]}{ }^{\rho, \sigma \lambda}+\partial_{[\mu} \chi_{\nu]}^{\rho, \sigma \lambda}+\partial_{[\mu} \chi_{\nu]}^{\sigma \lambda,}{ }^{\rho}\right)=0,
\end{gathered}
$$

where we define $\hat{\omega}$ as

$$
\hat{\omega}_{\mu, \nu, \rho \sigma} \equiv \omega_{\mu,(\rho, \sigma) \nu}=\frac{1}{2} \omega_{\mu, \rho, \sigma \nu}+\frac{1}{2} \omega_{\mu, \sigma, \rho \nu} .
$$

The equation (34) follows from (33). Therefore, dynamical field equation of motion is (33). In order to make the equation (33) to be symmetric under permutations of indices $\nu, \rho, \sigma$ we should impose the following constraint on $\chi_{\mu \nu, \rho \sigma}$,

$$
\chi_{\mu \nu, \rho \sigma}=-\frac{1}{3} \omega_{[\mu \nu], \rho \sigma} .
$$

\footnotetext{
${ }^{6}$ Precisely speaking, this is not pure gauge because $\omega_{\mu, \nu, \rho \sigma}$ is also transformed under (iii).
} 
Imposing this constraint, we find that the equation (33) is symmetric under permutations of indices and is second order derivatives in $e_{\mu, \nu \rho}$.

Here, we summarize the constraints we imposed in this subsection as follows :

- Traceless constraints :

$$
e_{\mu, \rho}^{\rho}=0, \quad \omega_{\mu, \rho, \rho}^{\rho}=0
$$

- Field strength constraints :

$$
\begin{array}{r}
\partial_{[\mu} e_{\nu],}^{\rho \sigma}+\omega_{[\nu,}^{\rho, \sigma}{ }_{\mu]}=0, \\
\partial_{[\mu} \Omega_{\nu], \rho \sigma, \lambda \kappa}=0,
\end{array}
$$

where the constraint (39) can be solved as : $\Omega_{\mu, \rho \sigma, \lambda \kappa}=\partial_{\mu} \chi_{\rho \sigma, \lambda \kappa}$.

- Constraint on $\chi_{\mu \nu, \rho \sigma}$ :

$$
\chi_{\mu \nu, \rho \sigma}=-\frac{1}{3} \omega_{[\mu \nu], \rho \sigma}
$$

Imposing these constraints, the auxiliary fields $\omega_{\mu, \nu, \rho \sigma}$ and $\Omega_{\mu, \rho \sigma, \lambda \kappa}$ are expressed in terms of the dynamical field $e_{\mu, \nu \rho}$ and only a symmetric part remains in the equation of motion (33).

The constraints imposed on the spin-3 fields are the traceless constraints (37) and the field strength constraints (38), (39) and (40). These constraints have been imposed in order to express the equation (33) in terms of the dynamical field $e_{\mu_{1}, \mu_{2} \cdots \mu_{s}}$. However, viewed from the matrix model, field strengths should be introduced as independent degrees of freedom. There is a possibility that "the higher spin field strengths" propagate as asymmetric tensor fields.

\section{Gauge fixing}

So far, we analyzed the elimination of the extra degrees of freedom by imposing the constraints (37), (38), (39) and (40). Combining these constraints and the equation of motion (33) we find that the equation (33) is expressed in terms of second order derivatives in $e_{\mu, \nu \rho}$ and is symmetric under permutations of indices. However, these constraints cannot eliminate all extra degrees of freedom. The Fronsdal equation (18) is expressed in terms of the rank-3 totally symmetric tensor field $\phi_{\mu \nu \rho}$, but the equation (33) is expressed in terms of "the spin-3 vierbein" $e_{\mu, \nu \rho}$, which have the part that is not totally symmetric. Last remaining 
extra degrees of freedom is the part that is not totally symmetric in the indices of $e_{\mu, \nu \rho}$. Thus, we should eliminate this extra degrees of freedom and express dynamical variable in terms of $\phi_{\mu \nu \rho}$, in order to show that the equation (33) coincides with the Fronsdal equation (18). This can be done by fixing the gauge symmetries. Recall that there are three kinds of the gauge transformations (i), (ii) and (iii) and the gauge transformation (i) corresponds to the Fronsdal gauge transformation. Therefore it seems that we should fix the gauge symmetries (ii) and (iii). As we see, the gauge symmetries (ii) and (iii) can eliminate the part that is not totally symmetric in the indices of $e_{\mu, \nu \rho}$ and we can express dynamical variable in terms of the rank-3 totally symmetric tensor field : $\phi_{\mu \nu \rho}$.

First, we fix the gauge symmetry (iii). Gauge fixing can be done by transforming $\hat{\omega}_{\mu, \nu, \rho \sigma} \rightarrow$ $\hat{w}_{\mu, \nu, \rho \sigma}=\hat{\omega}_{\mu, \nu, \rho \sigma}+\Lambda_{\mu \nu, \rho \sigma}$, choosing the parameter $\Lambda_{\mu \nu, \rho \sigma}$ as

$$
\begin{aligned}
\Lambda_{\mu \nu, \rho \sigma} \equiv & -\hat{\omega}_{\mu, \nu, \rho \sigma}+\frac{1}{2} B_{\mu \nu, \rho \sigma}-\frac{1}{4}\left(B_{\mu \rho, \sigma \nu}+B_{\mu \sigma, \rho \nu}-B_{\rho \nu, \mu \sigma}-B_{\sigma \nu, \mu \rho}\right) \\
& -\beta \eta_{\mu \rho}\left(B_{\sigma, \nu}^{\prime}+B_{\nu, \sigma}^{\prime}\right)-\beta \eta_{\mu \sigma}\left(B_{\rho, \nu}^{\prime}+B_{\nu, \rho}^{\prime}\right) \\
& -\beta \eta_{\rho \nu}\left(B_{\mu, \sigma}^{\prime}+B_{\sigma, \mu}^{\prime}\right)-\beta \eta_{\sigma \nu}\left(B_{\mu, \rho}^{\prime}+B_{\rho, \mu}^{\prime}\right) \\
& +2 \beta \eta_{\mu \nu}\left(B_{\rho, \sigma}^{\prime}+B_{\sigma, \rho}^{\prime}\right)+2 \beta \eta_{\rho \sigma}\left(B_{\mu, \nu}^{\prime}+B_{\mu, \nu}^{\prime}\right)
\end{aligned}
$$

where we define $B_{\mu \nu, \rho \sigma}, B_{\mu, \nu}^{\prime}$ and $\beta$ as follows :

$$
\begin{aligned}
B_{\mu \nu, \rho \sigma} & \equiv \hat{\omega}_{\mu, \nu, \rho \sigma}-\hat{\omega}_{\nu, \mu, \rho \sigma} \\
& =\partial_{\mu} e_{\nu, \rho \sigma}-\partial_{\nu} e_{\mu, \rho \sigma} \\
B_{\mu, \nu}^{\prime} & \equiv B_{\mu, \rho,}{ }_{\nu}, \\
\beta & =\frac{1}{4(D-2)}
\end{aligned}
$$

$\Lambda_{\mu \nu, \rho \sigma}$ satisfies the following properties :

$$
\begin{aligned}
& \Lambda_{\mu \nu, \rho \sigma}=\Lambda_{\nu \mu, \rho \sigma}=\Lambda_{\mu \nu, \sigma \rho}, \quad \Lambda_{\mu(\nu, \rho \sigma)}=0 \\
& \Lambda_{\mu \rho,{ }_{\nu}{ }_{\nu}}=0, \quad \Lambda_{\mu \nu, \rho}{ }^{\rho}=0 .
\end{aligned}
$$

$\beta$ is determined by the traceless condition (46).

Next, we fix the gauge symmetry (ii) by transforming $e_{\mu, \nu \rho} \rightarrow \varepsilon_{\mu, \nu \rho}=e_{\mu, \nu, \rho}+\Lambda_{\mu, \nu \rho}$ with

$$
\Lambda_{\mu, \nu \rho} \equiv-e_{\mu, \nu \rho}+\phi_{\mu \nu \rho}+\alpha\left(\eta_{\mu \nu} \phi_{\rho}^{\prime}+\eta_{\mu \rho} \phi_{\nu}^{\prime}-2 \eta_{\nu \rho} \phi_{\mu}^{\prime}\right)
$$

$\Lambda_{\mu, \nu \rho}$ satisfies the following properties:

$$
\begin{aligned}
& \Lambda_{\mu, \nu \rho}=\Lambda_{\mu, \rho \nu}, \quad \Lambda_{(\mu, \nu \rho)}=0 \\
& \Lambda_{\mu, \rho}{ }^{\rho}=0
\end{aligned}
$$


where $\phi_{\mu}^{\prime} \equiv \phi_{\mu \rho}{ }^{\rho}, \alpha=\frac{1}{2(D-1)}$. $\alpha$ is determined by the traceless condition (49). Carrying out these transformation, we can remove the part that is not totally symmetric in the indices of $e_{\mu, \nu \rho}$ and we have $e_{\mu, \nu \rho}=\phi_{\mu \nu \rho}$. Substituting $\hat{w}_{\mu, \nu, \rho \sigma}$ and $\varepsilon_{\mu, \nu \rho}$ into the equation (33), we can show that the equation (33) coincides with the Fronsdal equation (18).

It is worth noting the relation between our formulation and the unfolded formalism of higher spin gauge field theory due to Vasiliev [13]. Vasiliev constructed free field Lagrangian using similar method to the one we have employed in this paper [5]. Only difference between the unfolded formalism and our formulation is the appearance of $\Omega_{\mu, \rho \sigma, \lambda \kappa}$ in free theory. In the unfolded formalism, $\Omega_{\mu, \rho \sigma, \lambda \kappa}$ appears in interacting theories and contribute to higher derivative interactions. It is interesting to investigate the relation between our formulation and the unfolded formalism by analyzing higher spin interactions.

\subsection{Free Spin- $s$ Equation in Flat Spacetime}

In this subsection, using the same method as the one we have employed in the previous subsection, we derive the free equation of motion of the rank- $s$ totally symmetric tensor field in $D$-dimensional flat spacetime (16) from that of the matrix model.

In order to deal with the spin-s case, we keep track of the $(s-1)$-th order homogeneous polynomial of the operators $\partial_{\mu}$ and $\varnothing_{\mu \nu}$ :

$$
\begin{aligned}
& A_{\mu}=i \partial_{\mu}+(i)^{s-1} e_{\mu},{ }^{\mu_{1} \cdots \mu_{s-1}} \partial_{\mu_{1}} \cdots \partial_{\mu_{s-1}} \\
& +\frac{(i)^{s-1}}{s-1} \omega_{\mu}{ }^{\mu_{1} \cdots \mu_{s-2}, \rho_{1} \sigma_{1}}\left\{\partial_{\mu_{1}} \cdots \partial_{\mu_{s-2}} \varnothing_{\rho_{1} \sigma_{1}}\right\} \\
& +\frac{(i)^{s-1}}{(s-1)(s-2)} \Omega_{\mu}{ }^{\mu_{1} \cdots \mu_{s-3}, \rho_{1} \sigma_{1}, \rho_{2} \sigma_{2}}\left\{\partial_{\mu_{1}} \cdots \partial_{\mu_{s-3}} \varnothing_{\rho_{1} \sigma_{1}} \varnothing_{\rho_{2} \sigma_{2}}\right\} \\
& +\frac{(i)^{s-1}}{(s-1)(s-2)(s-3)} \tilde{\Omega}_{(1), \mu}{ }^{\mu_{1} \cdots \mu_{s-4}, \rho_{1} \sigma_{1}, \rho_{2} \sigma_{2}, \rho_{3} \sigma_{3}}\left\{\partial_{\mu_{1}} \cdots \partial_{\mu_{s-4}} \varnothing_{\rho_{1} \sigma_{1}} \varnothing_{\rho_{2} \sigma_{2}} \varnothing_{\rho_{3} \sigma_{3}}\right\} \\
& +\frac{(i)^{s-1}}{(s-1) !} \tilde{\Omega}_{(s-3), \mu}^{\rho_{1} \sigma_{1}, \cdots, \rho_{s-1} \sigma_{s-1}}\left\{\emptyset_{\rho_{1} \sigma_{1}} \cdots \varnothing_{\rho_{s-1} \sigma_{s-1}}\right\} .
\end{aligned}
$$

$e_{\mu, \mu_{1} \cdots \mu_{s-1}}(x)$ is the "spin-s vierbein" and $\omega_{\mu, \mu_{1} \cdots \mu_{s-2}, \rho \sigma}(x)$ is "the spin-s connection". From the discussion in the previous subsection, it seems that $\tilde{\Omega}_{(i)}(i=1, \cdots, s-3)$ are not necessary to derive the equation of motion. We set these auxiliary fields to zero : $\tilde{\Omega}_{1}=\cdots=\tilde{\Omega}_{s-3}=0$. Therefore, $A_{\mu}$ becomes 


$$
\begin{aligned}
A_{\mu}=i \partial_{\mu} & +(i)^{s-1} e_{\mu}{ }^{\mu_{1} \cdots \mu_{s-1}} \partial_{\mu_{1}} \cdots \partial_{\mu_{s-1}} \\
& +\frac{(i)^{s-1}}{s-1} \omega_{\mu}{ }^{\mu_{1} \cdots \mu_{s-2}, \rho_{1} \sigma_{1}}\left\{\partial_{\mu_{1}} \cdots \partial_{\mu_{s-2}} \varnothing_{\rho_{1} \sigma_{1}}\right\} \\
& +\frac{(i)^{s-1}}{(s-1)(s-2)} \Omega_{\mu}{ }^{\mu_{1} \cdots \mu_{s-3}, \rho_{1} \sigma_{1}, \rho_{2} \sigma_{2}}\left\{\partial_{\mu_{1}} \cdots \partial_{\mu_{s-3}} \varnothing_{\rho_{1} \sigma_{1}} \varnothing_{\rho_{2} \sigma_{2}}\right\}
\end{aligned}
$$

Here we assume that the spin-s fields satisfy the traceless condition,

$$
e_{\mu, \mu_{1} \cdots \mu_{s-2} \rho}^{\rho}=0, \quad \omega_{\mu, \mu_{1} \cdots \mu_{s-2} \rho,{ }_{\sigma}}^{\rho}=0 .
$$

The rank-s symmetric tensor field $\phi_{\mu_{1} \cdots \mu_{s}}$ is defined as $\phi_{\mu_{1} \cdots \mu_{s}}=e_{\left(\mu_{1}, \mu_{2} \cdots \mu_{s}\right)} \cdot \phi_{\mu_{1} \cdots \mu_{s}}$ satisfies the double traceless condition $\phi_{\rho}^{\rho}{ }_{\rho}^{\sigma} \sigma \mu_{5} \cdots \mu_{s}=0$ as a consequence of the traceless condition (52).

Let us summarize the gauge transformations. In the case of spin-s, there are $s$ kinds of gauge transformation. Since auxiliary fields $\tilde{\Omega}_{(i)}(i=1, \cdots, s-3)$ are set to be zero, the following three kinds of gauge transformations remain :

(i) Generalized coordinate transformation, generated by $\Lambda=\lambda^{\mu_{1} \cdots \mu_{s-1}} \partial_{\mu_{1}} \cdots \partial_{\mu_{s-1}}$,

$$
\delta e_{\mu, \mu_{1} \cdots \mu_{s-1}}=\partial_{\mu} \lambda_{\mu_{1} \cdots \mu_{s-1}}, \quad \delta \omega_{\mu, \mu_{1} \cdots \mu_{s-2}, \mu_{s-1} \nu}=0, \quad \delta \Omega_{\mu, \mu_{1} \cdots \mu_{s-3}, \rho \sigma, \lambda \kappa}=0,
$$

where $\lambda_{\mu_{1} \cdots \mu_{s-1}}$ is symmetric under permutations of the indices $\mu_{1}, \cdots, \mu_{s-1}$.

(ii) Generalized local Lorentz transformation, generated by $\Lambda=\lambda^{\mu_{1} \cdots \mu_{s-2}, \rho \sigma}\left\{\partial_{\mu_{1}} \cdots \partial_{\mu_{s-2}} \varnothing_{\rho \sigma}\right\}$,

$$
\begin{aligned}
& \delta e_{\mu, \mu_{1} \cdots \mu_{s-1}}=\lambda_{\left(\mu_{1} \cdots \mu_{s-2}, \mu_{s-1}\right) \mu} \equiv \Lambda_{\mu, \mu_{1} \cdots \mu_{s-1}}, \\
& \delta \omega_{\mu, \mu_{1} \cdots \mu_{s-2}, \rho \sigma}=\partial_{\mu} \lambda_{\mu_{1} \cdots \mu_{s-2}, \rho \sigma}, \\
& \delta \Omega_{\mu, \mu_{1} \cdots \mu_{s-3}, \rho \sigma, \lambda \kappa}=0
\end{aligned}
$$

where $\lambda_{\mu_{1} \cdots \mu_{s-2}, \rho \sigma}$ is symmetric under permutations of the indices $\mu_{1}, \cdots, \mu_{s-2}$ and satisfies $\lambda_{\mu_{1} \cdots \mu_{s-2}, \rho \sigma}=-\lambda_{\mu_{1} \cdots \mu_{s-2}, \sigma \rho}$.

(iii) Auxiliary gauge transformation, generated by $\Lambda=\lambda_{\mu_{1} \cdots \mu_{s-3}, \rho \sigma, \lambda \kappa}\left\{\partial_{\mu_{1}} \cdots \partial_{\mu_{s-3}} \varnothing_{\rho \sigma} \varnothing_{\lambda \kappa}\right\}$,

$$
\begin{aligned}
& \delta e_{\mu, \mu_{1} \cdots \mu_{s-1}}=0, \\
& \delta \omega_{\mu, \mu_{1} \cdots \mu_{s-2}, \rho \sigma}=\lambda_{\left(\mu_{1} \cdots \mu_{s-3}, \mu_{s-2}\right) \mu, \rho \sigma}+\lambda_{\left(\mu_{1} \cdots \mu_{s-3}, \rho \sigma, \mu_{s-2}\right) \mu}, \\
& \delta \Omega_{\mu, \mu_{1} \cdots \mu_{s-3}, \rho \sigma, \lambda \kappa}=\partial_{\mu} \lambda_{\mu_{1} \cdots \mu_{s-3}, \rho \sigma, \lambda \kappa}
\end{aligned}
$$

where $\lambda_{\mu_{1} \cdots \mu_{s-3}, \rho \sigma, \lambda \kappa}$ is symmetric under permutations of the indices $\mu_{1}, \cdots, \mu_{s-3}$ and satisfies $\lambda_{\mu_{1} \cdots \mu_{s-3}, \rho \sigma, \lambda \kappa}=-\lambda_{\mu_{1} \cdots \mu_{s-3}, \sigma \rho, \lambda \kappa}=-\lambda_{\mu_{1} \cdots \mu_{s-3}, \rho \sigma, \kappa \lambda}$. 
Therefore, $\phi_{\mu_{1} \cdots \mu_{s}}=e_{\left(\mu_{1}, \mu_{2} \cdots \mu_{s}\right)}$ are transformed under (i) as

$$
\delta \phi_{\mu_{1} \cdots \mu_{s}}=\partial_{\left(\mu_{1}\right.} \lambda_{\left.\mu_{2} \cdots \mu_{s}\right)} .
$$

This is consistent with the identification $\phi_{\mu_{1} \cdots \mu_{s}}=e_{\left(\mu_{1}, \mu_{2} \cdots \mu_{s}\right)}$. In order to derive the equation of motion (16) from that of the matrix model, what we must do is to impose constraints on the auxiliary fields and fix the gauge symmetries (ii) and (iii), as in the spin-3 case.

\section{Constraints}

Constraints are imposed on the coefficients of $\partial^{s-1}$ and $\partial^{s-3} \emptyset^{2}$ in the commutator of $A_{\mu}$. We obtain

$$
\begin{aligned}
\partial_{[\mu} e_{\nu]}{ }^{\mu_{1} \cdots \mu_{s-1}}+\omega_{[\nu}{ }^{\mu_{1} \cdots \mu_{s-2}, \mu_{s-1}}{ }_{\mu]} & =0, \\
\partial_{[\mu} \Omega_{\nu], \mu_{1} \cdots \mu_{s-3}, \rho \sigma, \lambda \kappa} & =0 .
\end{aligned}
$$

The constraint (58) implies that $\Omega_{\mu, \mu_{1} \cdots \mu_{s-3}, \rho \sigma, \lambda \kappa}$ can be written as a "pure gauge" configuration:

$$
\Omega_{\mu, \mu_{1} \cdots \mu_{s-3}, \rho \sigma, \lambda \kappa}=\partial_{\mu} \chi_{\mu_{1} \cdots \mu_{s-3}, \rho \sigma, \lambda \kappa} .
$$

We impose constraints on $\chi_{\mu_{1} \cdots \mu_{s-3}, \rho \sigma, \lambda \kappa}$ as

$$
\chi_{\mu_{1} \cdots \mu_{s-3}, \rho \sigma, \lambda \kappa}=-\frac{1}{s} \omega_{[\rho, \sigma] \mu_{1} \cdots \mu_{s-3}, \lambda \kappa} .
$$

Imposing these constraints, the commutators of $A_{\mu}$ become

$$
\begin{aligned}
{\left[A_{\mu}, A_{\nu}\right]=\frac{(i)^{s-1}}{(s-1)}\left[\partial_{[\mu} \omega_{\nu]}{ }^{\mu_{1} \cdots \mu_{s-2}, \rho \sigma}-\Omega_{[\mu}{ }^{\mu_{1} \cdots \mu_{s-3}, \mu_{s-2}}{ }_{\nu]},{ }^{\rho \sigma}\right.} & \left.-\Omega_{[\mu}{ }^{\mu_{1} \cdots \mu_{s-3}, \rho \sigma, \mu_{s-2}}{ }_{\nu]}\right] \\
& \times\left\{\partial_{\mu_{1}} \cdots \partial_{\mu_{s-2}} \varnothing_{\rho \sigma}\right\}
\end{aligned}
$$

and

$$
\begin{aligned}
& {\left[A^{\mu},\left[A_{\mu}, A_{\nu}\right]\right]=-(i)^{s}\left[\partial_{[\mu} \omega_{\nu]}^{\mu_{1} \cdots \mu_{s-2}, \mu_{s-1} \mu}\right.} \\
& \left.\left.\left.-\Omega_{[\mu}{ }^{\mu_{1} \cdots \mu_{s-3}, \mu_{s-2}} \nu\right],{ }^{\mu_{s-1} \mu}-\Omega_{[\mu}{ }^{\mu_{1} \cdots \mu_{s-3}, \mu_{s-1} \mu, \mu_{s-2}} \nu\right]\right] \times\left\{\partial_{\mu_{1}} \cdots \partial_{\mu_{s-1}}\right\} \\
& +\frac{(i)^{s}}{s-1} \partial^{\mu}\left(\partial_{[\mu} \omega_{\nu]}{ }^{\mu_{1} \cdots \mu_{s-2}, \rho \sigma}\right. \\
& \left.\left.\left.-\Omega_{[\mu}^{\mu_{1} \cdots \mu_{s-3}, \mu_{s-2}} \nu\right],{ }^{\rho \sigma}-\Omega_{[\mu}^{\mu_{1} \cdots \mu_{s-3}, \rho \sigma, \mu_{s-2}} \nu\right]\right) \times\left\{\partial_{\mu_{1}} \cdots \partial_{\mu_{s-2}} \varnothing_{\rho \sigma}\right\} .
\end{aligned}
$$


Therefore we obtain the equations of motion

$$
\begin{aligned}
& \partial_{[\mu} \hat{\omega}_{\nu]}{ }^{\mu, \mu_{1} \cdots \mu_{s-2} \mu_{s-1}}-\Omega_{[\mu}{ }^{\left(\mu_{1} \cdots \mu_{s-3}, \mu_{s-2}\right.}{ }_{\nu]},{ }^{\left.\mu_{s-1}\right) \mu}-\Omega_{[\mu}{ }^{\left(\mu_{1} \cdots \mu_{s-3}, \mu_{s-1} \mu, \mu_{s-2}\right)}{ }_{\nu]}=0, \\
& \left.\partial^{\mu}\left(\partial_{[\mu} \omega_{\nu]}{ }^{\mu_{1} \cdots \mu_{s-2}, \rho \sigma}-\Omega_{[\mu}{ }^{\mu_{1} \cdots \mu_{s-3}, \mu_{s-2}} \nu\right],{ }^{\rho \sigma}-\Omega_{[\mu}{ }^{\mu_{1} \cdots \mu_{s-3}, \rho \sigma, \mu_{s-2}}{ }_{\nu]}\right)=0,
\end{aligned}
$$

where $\hat{\omega}_{\mu, \nu, \mu_{1} \cdots \mu_{s-1}}$ is defined as

$$
\hat{\omega}_{\mu, \nu, \mu_{1} \cdots \mu_{s-1}} \equiv \omega_{\mu,\left(\mu_{1} \cdots \mu_{s-2}, \mu_{s-1}\right) \nu} .
$$

The second equation (64) can be derived by using the first one (63). Therefore, dynamical field equation is the first one. Owing to the constraint (60), the equation of motion (63) are symmetric under permutations of indices.

Here, we summarize the constraints we imposed in this subsection as follows :

- Traceless constraints :

$$
e_{\mu, \mu_{1} \cdots \mu_{s-3} \rho}{ }^{\rho}=0, \quad \omega_{\mu, \mu_{1} \cdots \mu_{s-2} \rho,}{ }^{\rho} \sigma=0 .
$$

- Field strength constraints :

$$
\begin{aligned}
\left.\partial_{[\mu} e_{\nu]}{ }^{\mu_{1} \cdots \mu_{s-1}}+\omega_{[\nu,}{ }^{\mu_{1} \cdots \mu_{s-2}, \mu_{s-1}} \mu\right] & =0 \\
\partial_{[\mu} \Omega_{\nu], \mu_{1} \cdots \mu_{s-3}, \rho \sigma, \lambda \kappa} & =0 .
\end{aligned}
$$

The constraint (68) can be solved as

$$
\Omega_{\mu, \mu_{1} \cdots \mu_{s-3}, \rho \sigma, \lambda \kappa}=\partial_{\mu} \chi_{\mu_{1} \cdots \mu_{s-3}, \rho \sigma, \lambda \kappa}
$$

- Constraints on $\chi_{\mu_{1} \cdots \mu_{s-3}, \rho \sigma, \lambda \kappa}$ :

$$
\chi_{\mu_{1} \cdots \mu_{s-3}, \rho \sigma, \lambda \kappa}=-\frac{1}{s} \omega_{[\rho, \sigma] \mu_{1} \cdots \mu_{s-3}, \lambda \kappa} .
$$

Imposing these constraints, the auxiliary fields $\omega_{\mu, \mu_{1} \cdots \mu_{s-3}, \rho \sigma}$ and $\Omega_{\mu, \mu_{1} \cdots \mu_{s-3}, \rho \sigma, \lambda \kappa}$ are expressed in terms of the dynamical field $e_{\mu, \mu_{1} \cdots \mu_{s-1}}$ and only a symmetric part remains in the equation of motion (63).

\section{Gauge fixing}


First, we fix the gauge symmetry (iii) by transforming $\hat{\omega}_{\nu, \mu, \mu_{1} \cdots \mu_{s-1}} \rightarrow \hat{w}_{\nu, \mu, \mu_{1} \cdots \mu_{s-1}}=$ $\hat{\omega}_{\nu, \mu, \mu_{1} \cdots \mu_{s-1}}+\Lambda_{\nu \mu, \mu_{1} \cdots \mu_{s-1}}$, choosing the parameter $\Lambda_{\nu \mu, \mu_{1} \cdots \mu_{s-1}}$

$$
\begin{aligned}
\Lambda_{\nu \mu, \mu_{1} \cdots \mu_{s-1}}= & -\omega_{\nu, \mu, \mu_{1} \cdots \mu_{s-1}}+\frac{1}{2} B_{\nu \mu, \mu_{1} \cdots \mu_{s-1}} \\
& -\frac{1}{2}\left\{B_{\nu \mu_{1}, \mu \mu_{2} \cdots \mu_{s-1}}+B_{\mu \mu_{1}, \nu \mu_{2} \cdots \mu_{s-1}}\right\}_{\mu_{i}} \\
& +\beta_{1} \eta_{\nu \mu}\left\{B_{\mu_{1}, \mu_{2} \cdots \mu_{s-1}}^{\prime}\right\}_{\mu_{i}} \\
& +\beta_{2}\left\{\eta_{\nu \mu_{1}} B_{\mu, \mu_{2} \cdots \mu_{s-1}}^{\prime}+\eta_{\mu \mu_{1}} B_{\nu, \mu_{2} \cdots \mu_{s-1}}^{\prime}\right\}_{\mu_{i}} \\
& -\left(\beta_{1}+\beta_{2}\right)\left\{\eta_{\nu \mu_{1}} B_{\mu_{2}, \mu \mu_{3} \cdots \mu_{s-1}}^{\prime}+\eta_{\mu \mu_{1}} B_{\mu_{2}, \nu \mu_{3} \cdots \mu_{s-1}}^{\prime}\right\}_{\mu_{i}} \\
& -\beta_{2}\left\{\eta_{\mu_{1} \mu_{2}} B_{\nu, \mu \mu_{3} \cdots \mu_{s-1}}^{\prime}+\eta_{\mu_{1} \mu_{2}} B_{\mu, \nu \mu_{3} \cdots \mu_{s-1}}^{\prime}\right\}_{\mu_{i}} \\
& +\left(\beta_{1}+2 \beta_{2}\right)\left\{\eta_{\mu_{1} \mu_{2}} B_{\mu_{3}, \nu \mu \mu_{4} \cdots \mu_{s-1}}^{\prime}\right\}_{\mu_{i}},
\end{aligned}
$$

where \{\}$_{\mu_{i}}$ denotes the symmetrization of indices $\mu_{i}(i=1, \cdots, s-1)$. We define $B_{\mu \nu, \mu_{1} \cdots \mu_{s-1}}$, $B_{\mu_{1}, \mu_{2} \cdots \mu_{s-1}}^{\prime}, \beta_{1}$ and $\beta_{2}$ as follows :

$$
\begin{aligned}
B_{\mu \nu, \mu_{1} \cdots \mu_{s-1}} & \equiv \hat{\omega}_{\mu, \nu, \mu_{1} \cdots \mu_{s-1}}-\hat{\omega}_{\nu, \mu, \mu_{1} \cdots \mu_{s-1}} \\
& =\partial_{\mu} e_{\nu, \mu_{1} \cdots \mu_{s-1}}-\partial_{\nu} e_{\mu, \mu_{1} \cdots \mu_{s-1}}, \\
B_{\mu_{1}, \mu_{2} \cdots \mu_{s-1}}^{\prime} & \equiv B_{\mu_{1} \rho,}{ }^{\rho} \mu_{2} \cdots \mu_{s-1} \\
\beta_{1} & =\frac{s-2}{D-2} \\
\beta_{2} & =\frac{D(s-2)}{2(D-2)(3-s-D)}
\end{aligned}
$$

The parameter $\Lambda_{\nu \mu, \mu_{1} \cdots \mu_{s-1}}$ is symmetric under permutations of the indices $\mu_{1}, \cdots, \mu_{s-1}$ and satisfies the following properties :

$$
\begin{array}{cl}
\Lambda_{\nu \mu, \mu_{1} \cdots \mu_{s-1}}= & \Lambda_{\mu \nu, \mu_{1} \cdots \mu_{s-1}}, \\
\Lambda_{\nu \mu, \mu_{1} \cdots \mu_{s-3} \rho}{ }^{\rho}=0, & \Lambda_{\nu \rho,}{ }^{\rho}{ }_{\mu_{1} \cdots \mu_{s-2}}=0 .
\end{array}
$$

$\beta_{1}$ and $\beta_{2}$ are determined by the traceless conditions (77).

Next, we fix the gauge symmetry (ii) by transforming $e_{\mu_{1}, \mu_{2} \cdots \mu_{s}} \rightarrow \varepsilon_{\mu_{1}, \mu_{2} \cdots \mu_{s}}=e_{\mu_{1}, \mu_{2} \cdots \mu_{s}}+$ $\Lambda_{\mu_{1}, \mu_{2} \cdots \mu_{s}}$ with

$$
\Lambda_{\mu_{1}, \mu_{2} \cdots \mu_{s}}=-e_{\mu_{1}, \mu_{2} \cdots \mu_{s}}+\phi_{\mu_{1} \cdots \mu_{s}}+\alpha \eta_{\mu_{1}\left(\mu_{2}\right.} \phi_{\left.\mu_{3} \cdots \mu_{s}\right)}^{\prime}-\alpha \eta_{\left(\mu_{2} \mu_{3}\right.} \phi_{\left.\mu_{4} \cdots \mu_{s}\right) \mu_{1}}^{\prime},
$$

where $\alpha=\frac{(s-1)(s-2)}{2(s+D-4)} \cdot \Lambda_{\mu_{1}, \mu_{2} \cdots \mu_{s}}$ is symmetric under permutations of indices $\mu_{2}, \cdots, \mu_{s}$ and satisfies the traceless condition

$$
\Lambda_{\mu_{1}, \mu_{2} \cdots \mu_{s-2} \rho} \rho=0 .
$$


Carrying out these transformation, we can remove the part that is not totally symmetric in the indices of $e_{\mu_{1}, \mu_{2} \cdots \mu_{s}}$ and we have $e_{\mu_{1}, \mu_{2} \cdots \mu_{s}}=\phi_{\mu_{1} \cdots \mu_{s}}$. Substituting $\hat{w}_{\nu, \mu, \mu_{1} \cdots \mu_{s-1}}$ and $\varepsilon_{\mu_{1}, \mu_{2} \cdots \mu_{s}}$ into the equation (63)), we can show that (63) coincides with the Fronsdal equation (16).

\section{Conclusions and Future Works}

In this paper, we have shown that the free equation of motion of bosonic massless higher spin gauge fields in $D$-dimensional flat spacetime can be derived from that of the matrix model based on the new interpretation of the matrix model. In order to derive higher spin field equations, we have done the two things : 1) imposing constraints 2) performing gauge fixing procedures. The results of this paper suggest that bosonic massless higher spin fields can be embedded in the matrix model. This is a first step towards construction of interacting massless higher spin gauge field theory by using the matrix model. Therefore, the matrix model can be used as a new approach to formulate massless higher spin gauge field theory.

There are several things which should be studied further. One is the derivation of free fermionic massless higher spin gauge field equations. Recently, it was shown that supergravity can be embedded in the supermatrix model [22]. There is a possibility that fermionic higher spin fields are embedded in the supermatrix model. Another one is to construct the interacting massless higher spin gauge field theory. As mentioned in Introduction, it is difficult to construct interacting theory. Difficulties associated with the requirement of gauge invariance can be overcome by using the matrix model because it has gauge invariance manifestly.

\section{Acknowledgments}

I am grateful to N.Ishibashi and K.Murakami for useful discussions. I also thank K.Araki, Y.Baba, N.Hatano and S.Katagiri for comments.

\section{References}

[1] M. Hanada, H. Kawai and Y. Kimura, "Describing curved spaces by matrices," Prog. Theor. Phys. 114, 1295 (2006) arXiv:hep-th/0508211. 
[2] C. Fronsdal, "Massless Fields With Integer Spin," Phys. Rev. D 18, 3624 (1978).

[3] J. Fang and C. Fronsdal, "Massless Fields With Half Integral Spin," Phys. Rev. D 18, 3630 (1978).

[4] B. de Wit and D. Z. Freedman, "Systematics Of Higher Spin Gauge Fields," Phys. Rev. D 21, 358 (1980).

[5] M. A. Vasiliev, "'Gauge' Form Of Description Of Massless Fields With Arbitrary Spin," Yad. Fiz. 32, 855 (1980), Sov. J. Nucl. Phys,32(3), 439 (1980).

[6] G. Velo and D. Zwanziger, "Propagation And Quantization Of Rarita-Schwinger Waves In An External Electromagnetic Potential," Phys. Rev. 186, 1337 (1969).

[7] C. Aragone and S. Deser, "Higher Spin Vierbein Gauge Fermions And Hypergravities," Nucl. Phys. B 170, 329 (1980). C. Aragone and S. Deser, "Consistency Problems Of Spin-2 Gravity Coupling," Nuovo Cim. B 57, 33 (1980). C. Aragone and S. Deser, "Consistency Problems Of Hypergravity," Phys. Lett. B 86, 161 (1979).

[8] F. A. Berends, J. W. van Holten, B. de Wit and P. van Nieuwenhuizen, "On Spin 5/2 Gauge Fields," J. Phys. A 13, 1643 (1980).

[9] C. Aragone and H. La Roche, "Massless Second Order Tetradic Spin 3 Fields And Higher Helicity Bosons," Nuovo Cim. A 72, 149 (1982).

[10] A. K. H. Bengtsson and I. Bengtsson, "Massless Higher Spin Fields Revisited," Class. Quant. Grav. 3, 927 (1986).

[11] F. A. Berends, G. J. H. Burgers and H. Van Dam, 'On Spin Three Selfinteractions," Z. Phys. C 24, 247 (1984). F. A. Berends, G. J. H. Burgers and H. van Dam, "On The Theoretical Problems In Constructing Interactions Involving Higher Spin Massless Particles," Nucl. Phys. B 260, 295 (1985).

[12] D. Sorokin, "Introduction to the classical theory of higher spins," AIP Conf. Proc. 767, 172 (2005) arXiv:hep-th/0405069.

[13] X. Bekaert, S. Cnockaert, C. Iazeolla and M. A. Vasiliev, "Nonlinear higher spin theories in various dimensions," arXiv:hep-th/0503128. 
[14] E. Sezgin and P. Sundell, "Massless higher spins and holography," Nucl. Phys. B 644, 303 (2002) [Erratum-ibid. B 660, 403 (2003)] arXiv:hep-th/0205131.

[15] E. S. Fradkin and M. A. Vasiliev, "On The Gravitational Interaction Of Massless Higher Spin Fields," Phys. Lett. B 189, 89 (1987). E. S. Fradkin and M. A. Vasiliev, "Cubic Interaction In Extended Theories Of Massless Higher Spin Fields," Nucl. Phys. B 291, 141 (1987).

[16] S. Ouvry and J. Stern, 'Gauge Fields Of Any Spin And Symmetry," Phys. Lett. B 177, 335 (1986).

[17] A. K. H. Bengtsson, "A Unified Action For Higher Spin Gauge Bosons From Covariant String Theory," Phys. Lett. B 182, 321 (1986). A. K. H. Bengtsson, "Brst Approach To Interacting Higher Spin Gauge Fields," Class. Quant. Grav. 5, 437 (1988).

[18] X. Bekaert, N. Boulanger and S. Cnockaert, "Spin three gauge theory revisited," JHEP 0601, 052 (2006) arXiv:hep-th/0508048. N. Boulanger, S. Leclercq and S. Cnockaert, "Parity violating vertices for spin-3 gauge fields," Phys. Rev. D 73, 065019 (2006) arXiv:hep-th/0509118.

[19] I. L. Buchbinder and V. A. Krykhtin, "BRST approach to higher spin field theories," arXiv:hep-th/0511276.

[20] A. Sagnotti and M. Tsulaia, "On higher spins and the tensionless limit of string theory," Nucl. Phys. B 682, 83 (2004) arXiv:hep-th/0311257.

[21] N. Ishibashi, H. Kawai, Y. Kitazawa and A. Tsuchiya, "A large-N reduced model as superstring," Nucl. Phys. B 498, 467 (1997) arXiv:hep-th/9612115.

[22] M. Hanada, H. Kawai and Y. Kimura, "Curved superspaces and local supersymmetry in supermatrix model," arXiv:hep-th/0602210. 